\title{
The Research on Position and Orientation Constraint of Rootless Redundant Robots Based on Dynamic Modeling
}

\author{
LI Na ${ }^{1, a^{*}}$, JIANG Haiyong ${ }^{1, b}$, XING Yazhou ${ }^{1, c}$ and ZHANG Xianpeng ${ }^{1, d}$ \\ ${ }^{1}$ Department of Mechanical \& Electrical Engineering, Hebei Agricultural University, China \\ ares_lina@126.com, broejhy@126.com, 'xyz@hebau.edu.cn, Gray_zhang5@163.com
}

\begin{abstract}
Keywords: Rootless redundant robots, dynamics, locomotion control, second-order nonholonomic, nominal mechnism

Abstract. Based on a Dynamic Nominal Mechanism adding to the rootless redundant robots, the dynamic modeling for can be established. The DNM is an idealized dynamic chain whose base is fixed at a point on earth, which makes the rootless redundant robots convert into the open chain multi-body system with fixed base. According to the established dynamic modeling, and the second-order nonholonomic constraints equations are developed from decomposition of the inertia matrix and the Christoffel tensor, and the position and orientation constraint is obtained then, which provides a research basis for designing the motion controller of rootless redundant robots. And the validity of the constraint equation is proved through the simulations of dynamic modeling of $8 \mathrm{R}$ rootless redundant robot.
\end{abstract}

\section{Introduction}

Comparing with other type of mobile robots (like wheeled robots, legged robots), the rootless redundant robots have advantages with higher adaptability to environment, hence this type of robots play an important role in many programs ${ }^{[1]}$, such as fire fighting, earthquake rescue, battlefield scouting. But because of the nonholomic constraint characteristic (without fixed base), it is difficult to control the position of the robots only through kinematic modeling. Therefore, researchers working on the problem of the rootless robot dynamic modeling has yet to be addressed ${ }^{[2,3]}$. The existing modeling methods of rootless redundant robots include Lagrange, Newton-Euler, and the application of product-of-exponentials formula ${ }^{[4-6]}$. Most of these researches on dynamic modeling are focused on the shape control, influence of environmental parameters and operating dynamics ${ }^{[7,8]}$, but the analysis on the position and orientation control of rootless redundant robots are seldom mentioned ${ }^{[9]}$.

This paper invetigates the position and orientation control of rootless redundant robots by using the concept of a Nominal Mechanism ${ }^{[10]}$ for the dynamic modeling. Based on this mehthod, planar motion of multi-rigid body system can be equivalent to open chain planar mechanism. According to the estiblished dynamic modeling, the second-order nonholonomic constraints equations are developed from decomposition of the inertia matrix and the Christoffel tensor, and the position and orientation constraint of the robots is obtained then. The kinematic parameters of position and oritentatoion are investigated during the simulation of a rootless redudant robots twith $8 \mathrm{R}$ joints.

\section{Dynamic nominal mechanism for the rootless redundant robots}

A rootless redundant robot consists of $n$ links connected with $n-1$ joints, which has $n+2$ degrees of freedom because there is connection between the base and the system. Generally, the coordinate of the first or last body, $\left(\boldsymbol{x}_{\mathrm{h}}, \boldsymbol{y}_{\mathrm{h}}\right)$, the angle $\phi_{\boldsymbol{h}}$ with inertia coordinate $x$ axis and each angle $\theta_{\boldsymbol{i}}$ $(\boldsymbol{i}=1,2, \cdots, \boldsymbol{n}-1)$ between the links, are taken as generalized coordinate of the system. In this paper, a nominal mechanism is designed, including two rotational joints and a prismatic joint between the end of the system and the base, which forms the open-chain multi-body system with RPR dynamic nominal mechanism shown in figure 1. Let the mass and inertia of the nominal mechanism is zero, which makes sure the nominal mechanism have no effect on the system. 


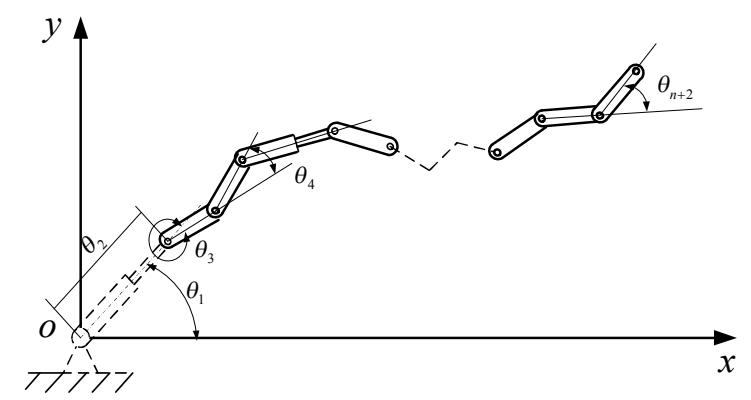

Fig. 1 Planar open chaining multi-rigid-body system and the nominal mechanism RPR

Based on nominal mechanism, the rootless open chain multi-body system is equivalent to be a planar open chain mechanism $\left(\mathrm{R}_{1} \mathrm{P}_{2} \mathrm{R}_{3}\right) \mathrm{R}_{4} \cdots \mathrm{R}_{n+2}$ with $n+2$ links. Take $\theta_{1}$ as the angle between the first nominal link with $x$ axis, and $\theta_{2}$ as the length between the nominal $\mathrm{R}_{1}$ to $\mathrm{R}_{3}$, and $\theta_{\boldsymbol{i}}(\boldsymbol{i}=3, \cdots \boldsymbol{n}+2)$ as the angles between link $i$ with link $i+1$.

\section{The dynamic molding and decomposition}

Based on the nominal dynamic mechanism, the rootless multi-rigid-body system can be translated to a underactuated open-chain multi-rigid-body mechanism with a fixed base. According to the influence coefficient method and virtual work principles ${ }^{[1]}$, the dynamic model of the rootless multi-rigid-body system can be described as following.

$$
\boldsymbol{M} \ddot{\boldsymbol{\theta}}+\dot{\boldsymbol{\theta}}^{T} \boldsymbol{C} \dot{\boldsymbol{\theta}}-\sum_{\boldsymbol{k}=1}^{\boldsymbol{n}+2} \boldsymbol{G}_{\boldsymbol{k}}^{T}\left(\boldsymbol{F}_{\boldsymbol{k}}^{\boldsymbol{g}}+\boldsymbol{F}_{\boldsymbol{k}}^{\boldsymbol{f}}\right)=K\left(\boldsymbol{\theta}_{\boldsymbol{s}}-\boldsymbol{\theta}\right)^{\mathrm{T}}
$$

Where, Eq. 1 is consist of $n+2$ second-order differential equations. Because of no input as the control variable in the 3 front equations of the dynamic model, it is the nonholonomic constraint of the rootless robot, that is the position constraint, and the rest of $n-1$ equations reprenst the dynamic control of the robot. Therefore, these positon constraint equations can be obtained by decomposing the above dynamic equations.

Let. The generalized joint displacements can be decomposed into nominal and actual joints as $\boldsymbol{\theta}=\left(\boldsymbol{\theta}_{\boldsymbol{v}}, \boldsymbol{\theta}_{\boldsymbol{a}}\right)^{T}$, where $\boldsymbol{\theta}_{\boldsymbol{v}}=\left(\theta_{1}, d_{2}, \theta_{3}\right)^{T}, \boldsymbol{\theta}_{\boldsymbol{a}}=\left(\theta_{3}, \cdots, \theta_{\boldsymbol{n}+2}\right)^{T}$ is the generalized joint displacements of the nominal joints and the actual joints. Similarly, the angle velocity and the angular acceleration is represented as $\dot{\boldsymbol{\theta}}=\left(\dot{\boldsymbol{\theta}}_{\boldsymbol{v}}, \dot{\boldsymbol{\theta}}_{\boldsymbol{a}}\right)^{T}, \ddot{\boldsymbol{\theta}}=\left(\ddot{\boldsymbol{\theta}}_{\boldsymbol{v}}, \ddot{\boldsymbol{\theta}}_{\boldsymbol{a}}\right)^{T}$. Let $\boldsymbol{G}_{\boldsymbol{k} \boldsymbol{v}}=\left[\begin{array}{lll}\boldsymbol{g}_{\boldsymbol{k}, 1} & \boldsymbol{g}_{\boldsymbol{k}, 2} & \boldsymbol{g}_{\boldsymbol{k}, 3}\end{array}\right]$ and $\boldsymbol{G}_{\boldsymbol{k} \boldsymbol{a}}=\left[\begin{array}{lll}\boldsymbol{g}_{\boldsymbol{k}, 4} & \cdots & \boldsymbol{g}_{\boldsymbol{k}, \boldsymbol{n}+2}\end{array}\right]$ as the first order influence coefficient of the nominal joints and real joints. The inertia matrix can be decomposed by differentiating the nominal joints from the actual joints. This gives

$$
M=\sum_{k=1}^{n+2}\left(G_{k}^{T} N_{k} G_{k}\right)=\left[\begin{array}{ll}
M_{v v} & M_{v a} \\
M_{a v} & M_{a a}
\end{array}\right]
$$

Where, $\quad \boldsymbol{M}_{\boldsymbol{v} v}=\sum_{k=1}^{n+2}\left(\boldsymbol{G}_{\boldsymbol{k} v}^{T} \boldsymbol{N}_{\boldsymbol{k}} \boldsymbol{G}_{\boldsymbol{k}}\right)$ relates the nominal joint accelerations to nominal joint torques; $\boldsymbol{M}_{\boldsymbol{v} \boldsymbol{a}}=\sum_{\boldsymbol{k}=1}^{n+2}\left(\boldsymbol{G}_{\boldsymbol{k} \boldsymbol{v}}^{T} \boldsymbol{N}_{\boldsymbol{k}} \boldsymbol{G}_{\boldsymbol{k} \boldsymbol{a}}\right)$ relates the nominal joint accelerations to actual joint torques; $\boldsymbol{M}_{\boldsymbol{a} v}=\sum_{\boldsymbol{k}=1}^{n+2}\left(\boldsymbol{G}_{\boldsymbol{k} \boldsymbol{a}}^{T} \boldsymbol{N}_{\boldsymbol{k}} \boldsymbol{G}_{\boldsymbol{k} \boldsymbol{v}}\right)$ relates the actual joint accelerations to nominal joint torques; $\boldsymbol{M}_{a \boldsymbol{a}}=\sum_{\boldsymbol{k}=1}^{n+2}\left(\boldsymbol{G}_{\boldsymbol{k} \boldsymbol{a}}^{T} \boldsymbol{N}_{\boldsymbol{k}} \boldsymbol{G}_{\boldsymbol{k} \boldsymbol{a}}\right)$ relates the actual joint accelerations to actual joint torques; With the 
Christoffel tensor $\boldsymbol{C}$, in the previous section, the second term of Eq. 1 can be rewritten as

$$
\dot{\boldsymbol{\theta}}^{T} \boldsymbol{C} \dot{\boldsymbol{\theta}}=\sum_{\boldsymbol{k}=3}^{n+2} \sum_{j=1}^{n+2} \sum_{i=1}^{n+2}\left(\dot{\theta}_{i} \boldsymbol{G}_{\boldsymbol{k}}^{T} \boldsymbol{N}_{\boldsymbol{k}} \frac{\partial \boldsymbol{g}_{\boldsymbol{k}}}{\partial \boldsymbol{\theta}_{\boldsymbol{i}}} \dot{\theta}_{j}\right)
$$

Decomposing the above according to nominal and actual joints yields

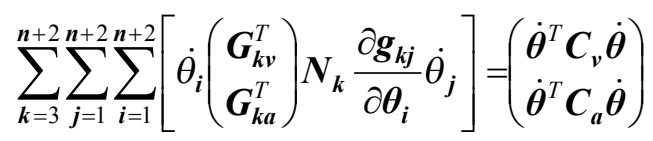

Where, $\boldsymbol{C}_{\boldsymbol{v}}=\sum_{\boldsymbol{k}=3}^{n+2}\left(\left(\boldsymbol{G}_{\boldsymbol{k} v}^{T} \boldsymbol{N}_{\boldsymbol{k}}\right) \otimes \boldsymbol{H}_{\boldsymbol{k}}\right)$ and $\boldsymbol{C}_{\boldsymbol{a}}=\sum_{\boldsymbol{k}=3}^{n+2}\left(\left(\boldsymbol{G}_{\boldsymbol{k} \boldsymbol{a}}^{T} \boldsymbol{N}_{\boldsymbol{k}}\right) \otimes \boldsymbol{H}_{\boldsymbol{k}}\right)$ are related the Christoffel tensor of the nominal joints to actual joints.

Further, the third term in Eq. 1 can be decomposed as

$$
\boldsymbol{\tau}^{f}=\left(\begin{array}{c}
\tau_{v}^{f} \\
\tau_{a}^{f}
\end{array}\right)=\sum_{k=3}^{n+2}\left(\begin{array}{l}
G_{k v}^{T} \\
G_{k a}^{T}
\end{array}\right)\left(F_{k}^{g}+F_{k}^{f}\right)
$$

Where, $\boldsymbol{\tau}_{v}^{f}=\sum_{k=3}^{n+2} \boldsymbol{G}_{k v}^{T}\left(\boldsymbol{F}_{k}^{g}+\boldsymbol{F}_{k}^{\boldsymbol{f}}\right)$ and $\boldsymbol{\tau}_{\boldsymbol{a}}^{\boldsymbol{f}}=\sum_{k=3}^{n+2} \boldsymbol{G}_{\boldsymbol{k} \boldsymbol{a}}^{T}\left(\boldsymbol{F}_{\boldsymbol{k}}^{\boldsymbol{g}}+\boldsymbol{F}_{\boldsymbol{k}}^{\boldsymbol{f}}\right)$

The last term in Eq. 1 can be decomposed into

$$
\boldsymbol{\tau}^{e}=\left(\begin{array}{c}
\boldsymbol{\tau}_{v}^{e} \\
\boldsymbol{\tau}_{a}^{e}
\end{array}\right)=\left(\begin{array}{cc}
K_{v} & 0 \\
0 & K_{a}
\end{array}\right)\left(\begin{array}{c}
\theta_{v s}-\theta_{v} \\
\theta_{a s}-\theta_{a}
\end{array}\right)
$$

Where the stiffness matrix is rearranged according to nominal and actual joints, and $K_{v}=0$.

Give the decoupling formulation for the nonholonomic open-chain multi-body system based on the above decomposition.

$$
\left(\begin{array}{ll}
M_{v v} & M_{v a} \\
M_{a v} & M_{a a}
\end{array}\right)\left(\begin{array}{l}
\ddot{\theta}_{v} \\
\ddot{\theta}_{a}
\end{array}\right)+\left(\begin{array}{l}
\dot{\theta}^{T} C_{v} \dot{\theta} \\
\dot{\theta}^{T} C_{a} \dot{\theta}
\end{array}\right)-\left(\begin{array}{c}
\tau_{v}^{f} \\
\tau_{a}^{f}
\end{array}\right)=\left(\begin{array}{c}
0 \\
K_{a}\left(\theta_{s a}-\theta_{a}\right)
\end{array}\right)
$$

Hence a nonholonomic constraints of the dynamic model of a rootless robot based on nominal mechanism is obtained:

$$
M_{v v} \ddot{\theta}_{v}+M_{v a} \ddot{\theta}_{a}+\dot{\theta}^{T} C_{v} \dot{\theta}-\tau_{v}^{f}=0
$$

Because the position (translation pair $\mathrm{P}_{2}$ ) and the attitude (revolute pair $\mathrm{R}_{1}$ and $\mathrm{R}_{3}$ ) of the multi-body system are described in the generalized coordinate supplied with nominal mechanism, Eq. 7 is the position-attitude constraint equation for the rootless robot.

\section{Position and orientation constraint of the rootless robot}

From the dynamic decomposition, it reveals the relationship between the accelerations of the nominal joints and the actuation force and between that and the centrifugal and Coriolis forces. In order to discuss the constraint characteristics, gravitational force is neglected and no external force is assumed resulting that $\tau_{v}^{f}=\tau_{a}^{f}=0$. From Eq. 6, the accelerations of actual joints are given as

$$
\ddot{\theta}_{a}=-\boldsymbol{M}_{a a}^{-1} \boldsymbol{M}_{a v} \ddot{\theta}_{v}-\boldsymbol{M}_{a a}^{-1} \dot{\theta}^{\mathrm{T}} \boldsymbol{C}_{a} \dot{\boldsymbol{\theta}}+\boldsymbol{M}_{a a}^{-1} K_{a}\left(\boldsymbol{\theta}_{a s}-\boldsymbol{\theta}_{a}\right)
$$


Substituting the above equation into the second-order constraint equation in Eq. 7 yields

$$
\ddot{\theta}_{v}=\boldsymbol{M}_{r v}^{-1}\left(\boldsymbol{M}_{v a} \boldsymbol{M}_{a a}^{-1} \dot{\theta}^{T} \boldsymbol{C}_{a} \dot{\boldsymbol{\theta}}-\dot{\boldsymbol{\theta}}^{T} \boldsymbol{C}_{\boldsymbol{v}} \dot{\boldsymbol{\theta}}+\boldsymbol{M}_{v a} \boldsymbol{M}_{a a}^{-1} K_{a}\left(\boldsymbol{\theta}_{a s}-\boldsymbol{\theta}_{a}\right)\right)
$$

Where, $\boldsymbol{M}_{r v}=\left(\boldsymbol{M}_{v v}-\boldsymbol{M}_{v a} \boldsymbol{M}_{a \boldsymbol{a}}^{-1} \boldsymbol{M}_{\boldsymbol{a} v}\right)^{-1}$ is an integrated control matrix. The above equation gives a dynamic coupling in terms of the acceleration of passive joints. The first two terms of the right-hand side of the above equation can be represented as $\dot{\boldsymbol{\theta}}^{\mathrm{T}} \boldsymbol{C}_{\boldsymbol{V} \boldsymbol{A}} \dot{\boldsymbol{\theta}}$, where, $\boldsymbol{C}_{\boldsymbol{V} \boldsymbol{A}}=\boldsymbol{C}_{\boldsymbol{v} \boldsymbol{v}}+\boldsymbol{C}_{\boldsymbol{v} \boldsymbol{a}}$, $\boldsymbol{C}_{\boldsymbol{v} v}=\sum_{\boldsymbol{k}=1}^{n+2}\left[\left(\boldsymbol{M}_{r v}^{-1} \boldsymbol{G}_{\boldsymbol{k} \boldsymbol{u}}^{\mathrm{T}} \boldsymbol{N}_{\boldsymbol{k}}\right) \otimes \boldsymbol{H}_{\boldsymbol{k}}\right], \quad \boldsymbol{C}_{\boldsymbol{v} \boldsymbol{a}}=\sum_{\boldsymbol{k}=1}^{n+2}\left[\left(-\boldsymbol{M}_{\boldsymbol{r} v}^{-1} \boldsymbol{M}_{\boldsymbol{v} \boldsymbol{a}} \boldsymbol{M}_{\boldsymbol{a} \boldsymbol{a}}^{-1} \boldsymbol{G}_{\boldsymbol{k} \boldsymbol{a}}^{\mathrm{T}} \boldsymbol{N}_{\boldsymbol{k}}\right) \otimes \boldsymbol{H}_{\boldsymbol{k}}\right]$.

Further, the coefficient of the third terms of the right-hand side of Eq. 9,

$$
\boldsymbol{M}_{c r}=\boldsymbol{M}_{r v}^{-1} \boldsymbol{M}_{v a} \boldsymbol{M}_{a a}^{-1} K_{a}
$$

and the acceleration of the nominal joints can be rewritten as

$$
\ddot{\theta}_{v}=M_{c r}\left(\theta_{a s}-\theta_{a}\right)+\dot{\theta}^{\mathrm{T}} \boldsymbol{C}_{\mathrm{VA}} \dot{\boldsymbol{\theta}}
$$

Eq.11 gives a direct relation between the acceleration of nominal joints and the displacement inputs of actual joints, i.e., the relation between the motion of rootless robot and the input of joints.

\section{Simulation}

Let the rootless robot be composed of 9 modules, so $\boldsymbol{n}=8$, and the length $\boldsymbol{l}_{\boldsymbol{i}}=0.1 \mathrm{~m}$, the mass $\boldsymbol{m}_{\boldsymbol{i}}=0.1 \mathrm{~kg}$, the inertia $\boldsymbol{I}_{\boldsymbol{i}}=0.1 \mathrm{kgm}^{2}$, the stiffness of joints $\boldsymbol{k}_{\boldsymbol{i}}=10 \mathrm{kNm} / \mathbf{r a d}$. Let the joint $1,2,3$ represent the nominal jonits RPR, and jonit 8 to 11 represent the acutal jonits. Suppose the input of actual joints be $\theta_{i}(\boldsymbol{t})=\pi / 6 \sin (\pi \boldsymbol{t}+2 \pi / 3(\boldsymbol{i}-1)+\pi / 3)$, and the displacement $\theta_{i}$, velocity $\dot{\theta}_{i}$ and acceleration $\ddot{\theta}_{i}(i=1,2,3)$ of nominal jonits are shown in Fig. $2-4$, according the amplitude of the inputs. Therefore the continuous motion can be imitated based on the nominal mechanism of the rootless robot, which is illustrated in Fig. 5.

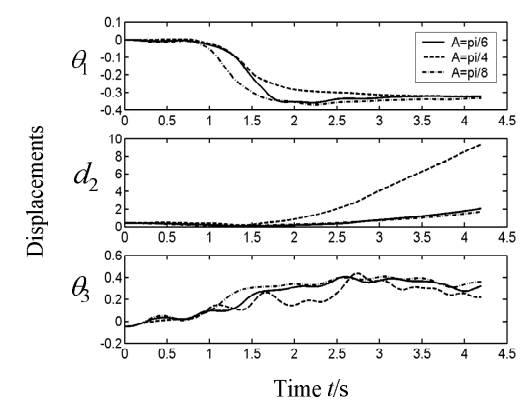

Fig.2 Displacements of the robot locomotion

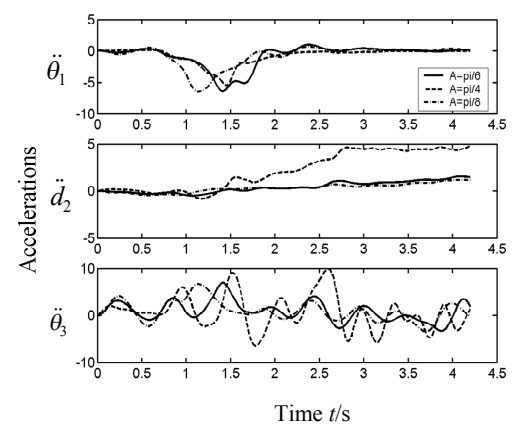

Fig.4 Accelerations of the robot locomotion

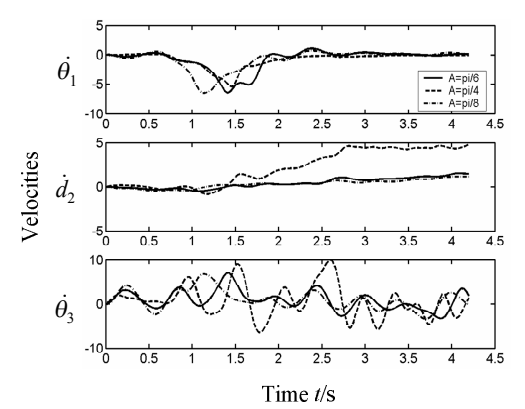

Fig.3 Velocities of the robot locomotion

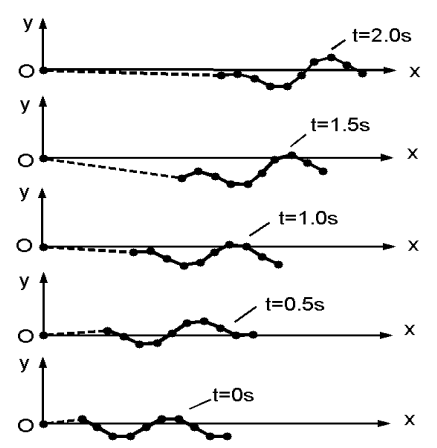

Fig.5 Animation of robot locmotion 
According to the illustrations above, the amplitude increase of the inputs has no influence on the attitude of the rootless robot, that is, the change of the angular displacement, angular velocity and angular acceleration of nominal mechanism is slight. Meanwhile, the influence on the liner displacement, velocity and acceleration is obvious, and the relationship is approximate positive proportion. Hence, the nominal mechanism can be taken in account as constraint condition for the description of position and attitude of the rootless robot.

\section{Conclusions}

This paper investigates the position and orientation control of rootless redundant robots by using the concept of a Nominal Mechanism for the dynamic modeling. The dynamic model provides a basis for analyzing the control of locomotion and manipulation of the robots. Depended on the dynamic modeling, the second order nonholonomic constraint equations were hence established by the decomposition of the dynamic formulation. And the motion acceleration constraint equation of the rootless redundant robots was obtained, which can be directly developed for forward and inverse dynamic locomotion control without complicated derivative or tedious calculations. The preliminary simulation results show that the position and orientation constraint based on dynamic modeling provides a research basis for designing the motion controller of rootless redundant robots.

\section{Acknowledgement}

This research is supported by the Natural Science Fund Committee of China 51305125 and Natural Science Foundation of Hebei Province E2013204110 and Excellent Youth Foundation of Hebei Province College Technology Committee YQ2013007.

\section{References}

[1] S. Hirose. Biologically Inspired Robots. Oxford: Oxford University Press, 1993. 1-49.

[2] F., Andre, D. Souza, L. Gadelha, Contact dynamics model of a space robotic manipulator, International Astronautical Federation 55th International Astronautical Congress 2004, 2: 777-785.

[3] P. LiljebÄack, O. Stavdahl, K. Pettersen. Modular pneumatic snake robot: 3D modelling, implementation and control. Model Ident Contr, 2008, 29: 21-28

[4] Transeth A, Van De Wouw N, Pavlov A, et al. Tracking control for snake robot joints. In: Proceedings of IEEE/RSJ International Conference on Intelligent Robots and Systems. San Diego: IEEE, 2007. 3539-3546

[5] Andersson S. Discretization of a continuous curve. IEEE Trans Robot, 2008, 24: 456-461

[6] McIsaac, K.A., Ostrowski, J.P. Motion Planning for Anguilliform Locomotion. IEEE Transaction on Robotics and Automation, pp. 637 - 652. IEEE Press, New York (2003)

[7] Ma S. Analysis of creeping locomotion of a snake-like robot. Adv Robot, 2001, 15: 205-224.

[8] Saito M, Fukaya M, Iwasaki T. Serpentine locomotion with robotic snakes. IEEE Contr Syst Mag, 2002, 22: 64-81.

[9] Z.F. Wang, S. Ma, B. Li, Y. Wang. A Unified dynamic model for locomtion and manipulation of a snake-like robot based on differentialgeometry. Science China, 2011, 41(2): 190-206.

[10] Zhao T, Li N. Nominal mechanism method of dynamic modeling for snake-like robots. Chin J Mech Eng, 2007, 43: 66-71. 